\title{
A dual-constriction biological nanopore
}

\author{
Addition of a second constriction to CsgG-family nanopores improves the \\ accuracy of homopolymer sequencing.
}

$\mathrm{N}$ anopore sequencing, a DNA sequencing technology originally proposed almost three decades ago, continues to evolve. It is based on the principles of nanopore sensing, the accurate measurement of a flow of ions through a nanometer-scale pore embedded inside a membrane. This flow of ions is obstructed when large molecules such as DNA pass through the pore. The current varies as different parts of the molecule, such as different DNA nucleotides, pass through, and can be used to 'read' or sequence the DNA. Researchers from Vrije Universiteit Brussel (VUB), in collaboration with Oxford Nanopore Technologies Ltd., have recently improved the ability to resolve the sequencing of homopolymer regions, a known cause of inaccuracies in nanopore sequencing, through the introduction of additional structural features in the nanopore design.

A structural constriction in the nanopore protein influences the current flowing through the channel. When a stretch of DNA sequence containing multiple consecutive nucleotides of the same type passes through the constriction, the signal becomes information-poor, making it difficult to determine the exact number of bases in this homonucleotide region. Addition of a second constriction at a fixed distance from the primary constriction could provide more sequence information (a principle alluded to by D. Stoddart et al. Angew. Chem. 49, 566-569, 2010). To explore this possibility, the researchers solved the cryo-electron microscopy structure of the CsgG nanopore protein in complex with its secretion partner CsgF to understand how CsgF interacts with CsgG. "To make the dual-constriction nanopores for DNA sequencing, we combined CsgG-derived nanopores with small fragments of CsgF that correspond only to the region making the second constriction (the N-terminal CsgF constriction peptide or FCP). The combined CsgG-FCP nanopores proved very stable and able to pass DNA similar to their CsgG parents," Han Remaut from VUB explains.

The researchers provide a proof-of-principle prototype for the dual-constriction sequencing nanopore and demonstrate a $25-70 \%$ improvement of single-read accuracy in homopolymer

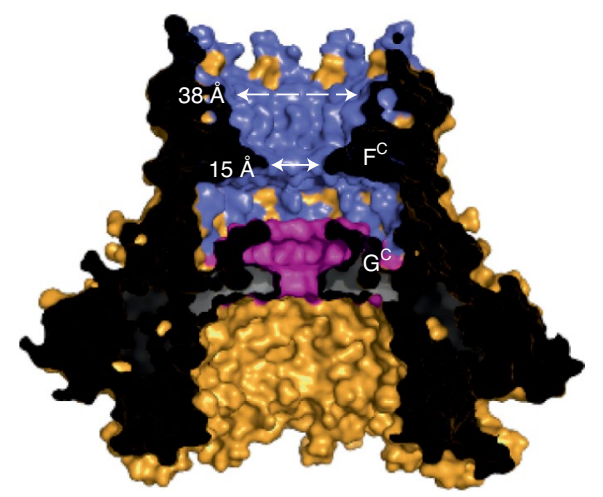

Cryo-electron microscopy structure of the CsgGCsgF pore shown in cross-sectional side view, depicted as solvent-accessible surface. GC, CsgG constriction; FC, CsgF constriction. Reprinted with permission from Nat. Biotechnol. https://doi. org/10.1038/s41587-020-0570-8, Springer Nature.

stretches up to nine nucleotides long, over that of the single-constriction CsgG nanopore. The current prototype uses wild-type CsgF fragments that have not been fully optimized for the quality of electrical signal obtained from the second constriction, and the researchers are interested in further engineering this system to improve performance.

When asked about the future of the nanopore sensing technology and the field, Remaut says, "DNA and RNA sequencing have been the biggest marketed biotech achievements of the nanopore field. There are several studies that show also the value of protein nanopores for small-molecule sensing and protein analysis. I look forward to the field further developing and translating this potential into real live applications. Dual constriction pores may also have advantages in these areas."

Arunima Singh

Published online: 1 September 2020 https://doi.org/10.1038/s41592-020-0949-y

Research paper

Van der Verren, S. E. et al. A dual-constriction biological nanopore resolves homonucleotide sequences with high fidelity. Nat. Biotechnol. https:// doi.org/10.1038/s41587-020-0570-8 (2020). 